\title{
Moving Object in Different Environment (MODE) using Spatial-Temporal Database Concept
}

\author{
V. Karthikeyani \\ $\mathrm{PhD}$ \\ Assistant Professor, \\ Department of \\ Computer Science, \\ Thiruvalluvar \\ Government Arts \\ College,Rasipuram, \\ India
}

\author{
I.Shahina Begam \\ Assistant Professor, \\ Department of \\ Computer Science, \\ Ratankanwar \\ Bhawarlal Gothi Jain \\ College for \\ Women, Chennai- \\ 600052,Tamilnadu, \\ India
}

\author{
K. Tajudin \\ Assistant Professor, \\ Department of \\ Computer Science, \\ The New College, \\ Royapettah, \\ Chennai- \\ 600014,Tamilnadu, \\ India
}

\author{
I.Parvin Begum \\ Lecturer, \\ Department of \\ Computer Science, \\ Soka Ikeda College \\ of Arts and \\ Science, Chennai- \\ 99,Tamilnadu, India
}

\begin{abstract}
Spatial-temporal data models and query languages are a topic of growing interest. A spatial-temporal database is a database that embodies spatial, temporal, and spatial-temporal database concepts and captures simultaneously spatial and temporal aspects of data. A spatial data object occupies a certain region of space, which is characterized by its location and boundary. It deals with geometries that change with time. In this research to focus the moving object in different environment with the algorithmic details of each object movement likewise sky, water fall and hills. The positions of moving objects are continuously changing over time. For modeling these moving objects, consider both continuous and discrete models. The position of moving object can be calculated by time, speed, route, hiding position and reflection angle etc. The speed and route are managed as dynamic attributes, and are the motion information of moving object. The attribute uses in whole concept in dynamic.
\end{abstract}

\section{Keywords}

Spatial Temporal Database-Moving Object- Hiding ValueLocation finding-Query analyzation

\section{INTRODUCTION}

There are two common models of spatial information: fieldbased and object-based. The field-based model treats spatial information such as altitude, rainfall and temperature as a collection of spatial functions transforming a space partition to an attribute domain. The spatial types and operations have been made to be a part of a query language such as SQL.

Most database systems model is the current state of the world, for instance, current customer, current students and courses currently being offered. In many applications, it is very important to store and retrieve information about past states. Databases that store information about states of the real world across time are called temporal databases.

In this research movement of object in different environment (MODE) is consider in point-based time model, moving points are positions or locations which changes accordingly. This section will describe MODE in MOST (Moving Object Spatio-Temporal) data model also. The algorithm will give knowledge about individual elements of the set represent the actual time instants, while the linear order represents the progression of time. The actual granularity of time can be chosen upon the application domain. The point-based models view the database as a sequence of snapshots.

$$
\text { mpoint: time -> point }
$$

That is, moving point is changed according to time. Query for certain time returns points which describes the existing position of moving object at that time. The query related to a moving point doesn't include a query about moving object's shape.

\section{LITERAL REVIEW}

Algorithm for moving object databases [1] described, first design a comprehensive system of data types and operations for moving objects (time dependent geometry) of an abstract model and finite representations for all the types of the abstract model that can be mapped into efficient data structure. The data type part of a discrete model. The algorithmic descriptions of lifted operation, originally defined for non-temporal objects now applied to moving variants of the arguments such as predicates, set operations, aggregation, numeric properties, distance and direction and Boolean operations.

A unified model for spatial and temporal information [2] was structured to progress from concrete motivating examples to a general theory. A model is then presented which focused ideas on purely spatial modeling involving two orthogonal dimensions representing database and event time. Next focused query algebra similar in relational algebra. The real time data used as network information and land ownership details.

Modeling historical and future spatio-temporal relationship of moving objects in databases [3,14,15] in this research moving object models have focused exclusively either on past or future movement. Balloon object is the first attempt to treat past and future movements of moving objects in a consistent and integrated manner. The balloon objects were defined as a temporal composition of the past and future movements of moving objects and queries. In this model to show the relationships between balloon objects are composed of certain and uncertain relationships between their parts.

In the previous research only considered the moving object 
in region wise and the object also using points, balloon etc. In this proposed work algorithmic consideration defines all the location movement of object and it's include different sizes of object. The reflection of the object in different angle is consider and hiding also.

\section{MODE ALGORITHM}

The MODE algorithms are described in three different ways. First consider the object movement in hill to discuss upwards, downwards position and also discuss another object in backside movement that is hiding movement. Second consider the object movement in the waterfall here to describe the reflection angle based movement of object also straight movement all position changes. Third consider the object movement in the sky here to describe not only speed different but also consider the no signal point. All the movement of object stored in the database with effective attributes and dynamic values updating.

\subsection{Moving Object in the Hill}

This section discusses the object movement in the hill. There are two ways to move the object, first discusses about the objects movement in the upwards and downwards direction of the hill with the difference of speed. Second one is to discuss about the object moving from left to right which contains the details of the complete movement of the objects updated with the hiding values. Figure (1) shows the details of the object movement in the hill; the attributes are ob_id, $x, y$, height, width, speed, duration and remarks.

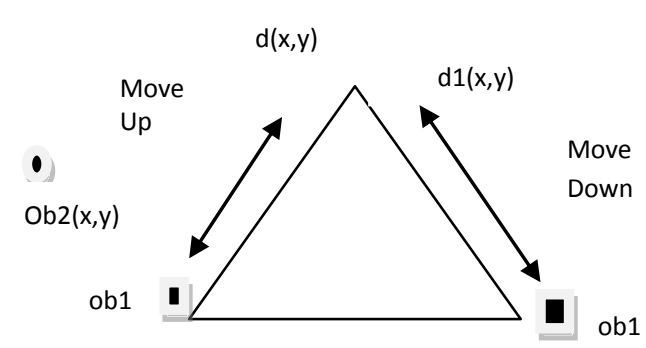

Fig.1: Object Movement in the Hill

In this section the algorithm discusses the details of the moving objects in the hill. The algorithm- 1 gives the details of object1 moving the hill from bottom to top, the parameter $\mathrm{x}$, $\mathrm{y}, \mathrm{w}$ and $\mathrm{h}$ pass the object movement. The step1 move the object from bottom to top, compare the value with the destination, first it checks the condition less then object1 $\mathrm{x}$ and $\mathrm{y}$ coordinate value with the destination $\mathrm{x}$ and $\mathrm{y}$ coordinate value. Next the $x$ coordinate increments value the y coordinate decrement of the value until the end of the loop. Once the value equal the destination $\mathrm{x}$ and $\mathrm{y}$ coordinate, stop the movement of the objects. The step 2 stores all the movements of the objects in the database. The step3 exits the algorithm.

Algorithm-1: ob1_MoveUpwards (int x, int y, int w, int h)

Variables: the value of $\mathrm{x}, \mathrm{y}, \mathrm{w}$ and $\mathrm{h}$ are left, top, width and height. Draw the object and hill, set the timer interval, move the object in the hill bottom to top, set the destination (d) value of the ob1.
1) The ob1 value compare with the destination (denote as d) value.

$$
\begin{aligned}
& \text { a) If ob1.x }<\text { d.x and ob1.y }>\text { d.y then } \\
& \text { For } \mathrm{i}=\text { ob1.y to d.top } \\
& \mathrm{xi}=\mathrm{xi}-1+\Delta \mathrm{x}(\Delta \mathrm{x}=\mathrm{xi}-\mathrm{xi}-1) \\
& \mathrm{yi}-1=\mathrm{yi}-\Delta \mathrm{y}(\Delta \mathrm{y}=\mathrm{yi}-\mathrm{yi}-1) \\
& \text { Move to step2 } \\
& \text { elseif ob1.x=d.x and ob1.y=d.y then }
\end{aligned}
$$

Return "stop the movement of the ob1"

Endif

2) Save all the values and remarks in the database until the value reaches the

End point.

3) Exit

The object movement in downwards also same pattern of algorithm-1 but will make the slight changes of speed and coordinates position increment just opposite of upwards movement.

The algorithm-2 object2 move from left to right (ob2_moveLtoR), set the destination value which is d2. The step1 is first object $2 \mathrm{x}$ and $\mathrm{y}$ coordinate value compare with the destination value. The condition is true, the loop continues until it reaches the destination, check the object $2 \mathrm{x}$ value with the hill(x, width) value and the object $2 \mathrm{y}$ value with the hill(y, height) value. The object in the hiding position increment the $\mathrm{x}$ coordinate value of the object but the $\mathrm{y}$ value is constant because the movement of the object is straight line or otherwise the $\mathrm{x}$ and $\mathrm{y}$ coordinate value incremented normally up to the destination. The step2 saves all the details of the object 2 movement in the hiding and normal movement. The step3 exits the algorithm.

\section{Algorithm-2: ob2_moveLtoR(int x, int y, int w, int h)}

Variables: the value of $\mathrm{x}, \mathrm{y}, \mathrm{w}$ and $\mathrm{h}$ are left, top, width and height. Draw the object and hill, set the timer interval, move the object from left to right, set the destination (d2) value of the ob2, d2.x and d2.y the coordinate value of the destination.

1) The ob2 value compare with the destination (denote as d) value.

a) If ob2.x $<\mathrm{d} 2 . x$ and ob2.y<d2.y then

For $\mathrm{i}=\mathrm{ob} 2 . \mathrm{x}$ to $\mathrm{d} 2 . \mathrm{x}$

If ob2.x $>=$ h.x and ob2.x $<=$ h.x + h.width and $o b 2 . y>=h . y$ and

$$
\text { ob2.y<= h.y + h.height then }
$$

(The object in the hiding position)

$$
\begin{aligned}
& \mathrm{x}_{\mathrm{i}}=\mathrm{x}_{\mathrm{i}-1}+\Delta \mathrm{x}\left(\Delta \mathrm{x}=\mathrm{x}_{\mathrm{i}}-\mathrm{x}_{\mathrm{i}-1}\right) \\
& \mathrm{y}_{\mathrm{i}}=\mathrm{y}_{\mathrm{i}-1}
\end{aligned}
$$

Move to step2

Else

$$
\mathrm{x}_{\mathrm{i}}=\mathrm{x}_{\mathrm{i}-1}+\Delta \mathrm{x}\left(\Delta \mathrm{x}=\mathrm{x}_{\mathrm{i}}-\mathrm{x}_{\mathrm{i}-1}\right)
$$


$\mathrm{y}_{\mathrm{i}}=\mathrm{y}_{\mathrm{i}-1}$

Move to step2

endif

elseif ob2. $x=d 2 . x$ and ob2.y $=\mathrm{d} 2 . y$ then

Return "stop the movement of the ob2"

Endif

2) Save all the values and remarks in the database until the value reaches the

End point.

3) Exit

Figure (1) shows the algorithm details explanation diagram of the moving object, ob1 $(\mathrm{x}, \mathrm{y})$ object moment from starting position to $\mathrm{d}(\mathrm{x}, \mathrm{y}), \mathrm{d} 1(\mathrm{x}, \mathrm{y})$ the same objectl moment in another direction with different speed to end with ob1 $(\mathrm{x}, \mathrm{y})$ bottom of the hill.

The value of the distance and duration of hiding location finding as(Table 1)

$$
\mathrm{H}_{\mathrm{d}}=\mathrm{H}_{\mathrm{e}}-\mathrm{H}_{\mathrm{s}}
$$
location

$\mathrm{H}_{\mathrm{d}}$ is the value of the distance covered in the hiding

$\mathrm{H}_{\mathrm{e}}, \mathrm{H}_{\mathrm{s}}$ - is the value of the starting and ending value of the hiding location

$$
\mathrm{H}_{\mathrm{t}}=\mathrm{T}_{\mathrm{e}}-\mathrm{T}_{\mathrm{s}}
$$

$\mathrm{H}_{\mathrm{t}}$ is the value of the duration in the hiding location

$\mathrm{T}_{\mathrm{e}}, \mathrm{T}_{\mathrm{s}}-$ is the value of the starting and ending duration of the hiding location

Table 1.Object Movement in the Hiding Location

\begin{tabular}{|c|c|}
\hline Object ID & Ob1 \\
\hline Average Speed & 10 (number of pixel) \\
\hline $\begin{array}{c}\text { Start Hide } \\
\text { Location }\end{array}$ & 4680 (pixel values) \\
\hline End Hide Location & $6720($ pixel values $)$ \\
\hline Start Duration & $4080(\mathrm{msec})$ \\
\hline End Duration & $6120(\mathrm{msec})$ \\
\hline
\end{tabular}

The value of the distance and duration of object move entire location (from left to right) as

$$
\mathrm{O}_{\mathrm{d}}=\mathrm{O}_{\mathrm{e}}-\mathrm{O}_{\mathrm{s}}
$$

$\mathrm{O}_{\mathrm{d}}$ is the value of the distance covered in the source to destination.

$\mathrm{O}_{\mathrm{e}}, \mathrm{O}_{\mathrm{s}}$ - is the value of the starting and ending value of the source to destination.

$$
\mathrm{O}_{\mathrm{t}}=\mathrm{T}_{\mathrm{e}}-\mathrm{T}_{\mathrm{s}}
$$
destination

$\mathrm{O}_{\mathrm{t}}$ is the value of the duration in the source to

$\mathrm{T}_{\mathrm{e}}, \mathrm{T}_{\mathrm{s}}-$ is the value of the starting and ending value of the source to destination

\subsection{Moving Object in the Waterfall}

The objects move in the waterfall with different type of movements. Generally the object movement is considered as the direction, speed of the object and location changes. The main concentration occur depends upon the above three basis. But in this section to cover all the three apply in the same object the different location and the different direction of movement.

The figure (2) shows object's movement in the waterfall. The figure describes the object movement in the three parts. The first part is the starting point of the object movement. here the object maximum in the hiding position. The second part of the object movement is the different changes making the object especially the distance, direction, speed and reflection etc. The object moves in the hiding position, it may be top movement in the straight line not possible to see the object in that movement. But the movement increases in the $\mathrm{x}$-coordinate value and slight modification occur in the $y$-coordinate value.

The object moves in downwards several times, in that situation to create the reflection of the object, the reflection angle appears depends upon the moving speed in the downwards direction. Suppose the angle of reflection may occur in $45^{\circ}$ the object speed is same as the reflection angle. Here to generate the $\mathrm{x}$ and $\mathrm{y}$ coordinate value in the time interval of $t_{i}$ and $t_{n}$ when the object in the reflection position.

$\mathrm{t}_{\mathrm{i}}$ duration the value of $\mathrm{x}_{\mathrm{i}}=\mathrm{x}_{\mathrm{i}-1}+\Delta \mathrm{x}\left(\Delta \mathrm{x}=\mathrm{x}_{\mathrm{i}}-\mathrm{x}_{\mathrm{i}-1}\right)$

$$
\mathrm{y}_{\mathrm{i}}=\mathrm{y}_{\mathrm{i}-1}-\Delta \mathrm{y}\left(\Delta \mathrm{y}=\mathrm{y}_{\mathrm{i}}-\mathrm{y}_{\mathrm{i}-1}\right)
$$

$t_{n}$ duration the value of $x_{n}=x_{n-1}+\Delta x\left(\Delta x=x_{n}-x_{n-1}\right)$

$$
\mathrm{y}_{\mathrm{n}}=\mathrm{y}_{\mathrm{n}-1}-\Delta \mathrm{y}\left(\Delta \mathrm{y}=\mathrm{y}_{\mathrm{n}}-\mathrm{y}_{\mathrm{n}-1}\right)
$$

The coordinate value of the $\mathrm{x}$ increases and the $\mathrm{y}$ value decreases when these values are repeated until the object moves in the downwards direction. $\Delta \mathrm{x}$ difference between current $\mathrm{x}$ coordinate and previous $\mathrm{x}$ coordinates value, $\Delta \mathrm{y}$ difference between current y coordinate and previous $\mathrm{y}$ coordinates value.

In the final stage the object moves in the straight line until it reaches the destination based on figure (2). In that movement the object $\mathrm{x}$-coordinate value increases and the y-coordinate value is same until it reaches the destination.

$$
\begin{aligned}
& t_{i} \text { duration the value of } x_{i}=x_{i-1}+\Delta x\left(\Delta x=x_{i}-x_{i-1}\right) \\
& y_{i}=y_{i-1} \\
& t_{n} \text { duration the value of } x_{n}=x_{n-1}+\Delta x\left(\Delta x=x_{n}-x_{n-1}\right) \\
& y_{n}=y_{n-1}
\end{aligned}
$$

The above eq(5) gives the details about the object increment in the $x$ and $y$ coordinates in the time interval of $t_{i}$ and $t_{n}$ when the object in the straight line final stage. 
In the algorithm-3 to give the details of when the object move in the reflection position. After the object downwards movement it's definitely get the reflection position. The step1 comparison is common for the entire algorithm, next check the object reflection angle assume the reflection angle $45^{\circ}$ depends on the flow of the object speed in the downwards movement. In that movement the value of $\mathrm{x}$ and $\mathrm{y}$ coordinate increases frequently, the nop set on the greatest value of the $\mathrm{dx}$ and dy value. The object reaches some of the distance its slightly down the speed and the angle will be change in the downwards flow. The speed is calculated as how much pixel passing the object for the particular interval, the tcount is calculated continuously from the starting to end position. The step2 save all the values of the object in the database. The step3 exit the object movement in the reflection position.

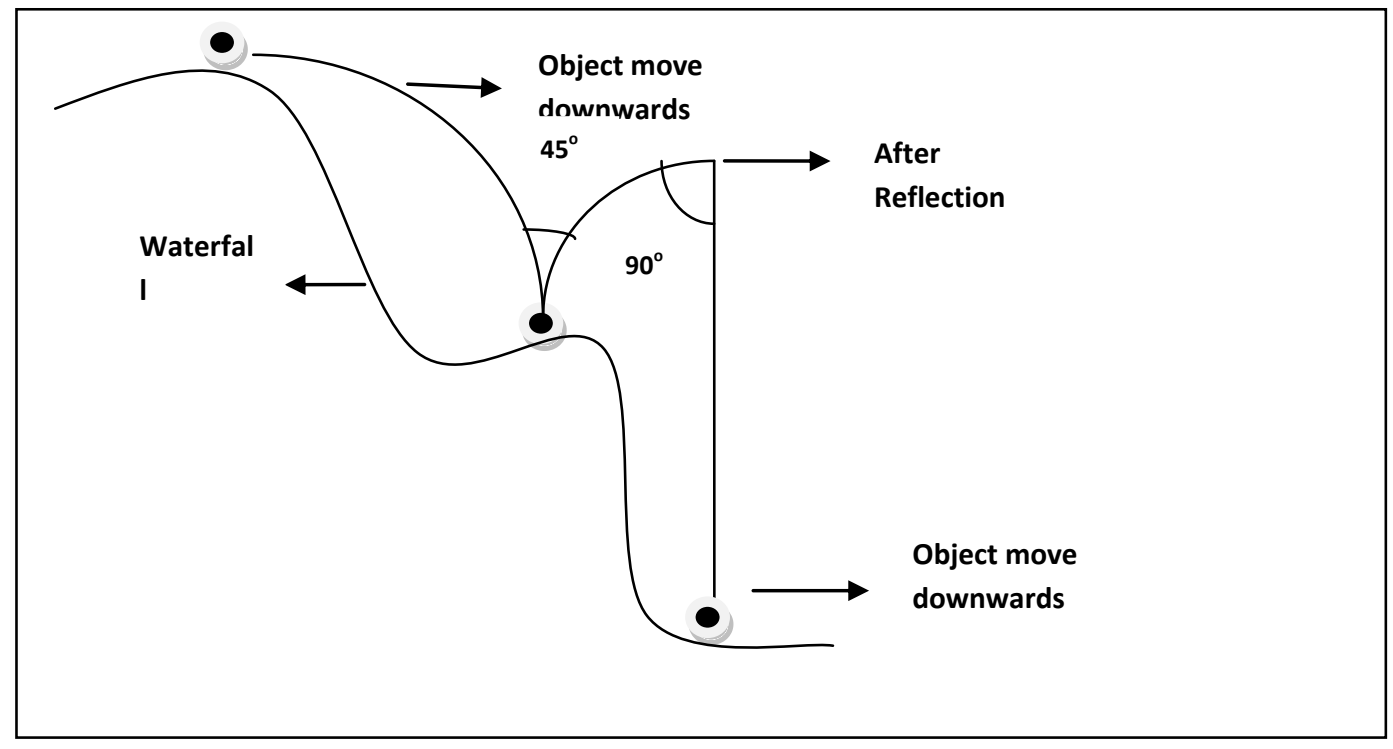

Fig.2: The Object Reflection in the Waterfall

The above figure (2) shows the movement of the object reflection in the waterfall.

\subsection{Moving Object in Sky}

The object movement in the sky is considering entire movement of the object flow. The object movement is considering the different speed; in the starting stage the movement in any environment is very slow, after some duration the speed slightly increases to the maximum level, finally reaching the destination.
The figure (3) shows the object movement in three stages. The first stage movement of the object in the starting condition, here the object movement is very slow, the second stage is the centre movement of the object here the object movement may be unknown, the third stage to reach the object after crossing the some unknown location move to the destination, in this stage the object movement to increase the speed until reach the destination.

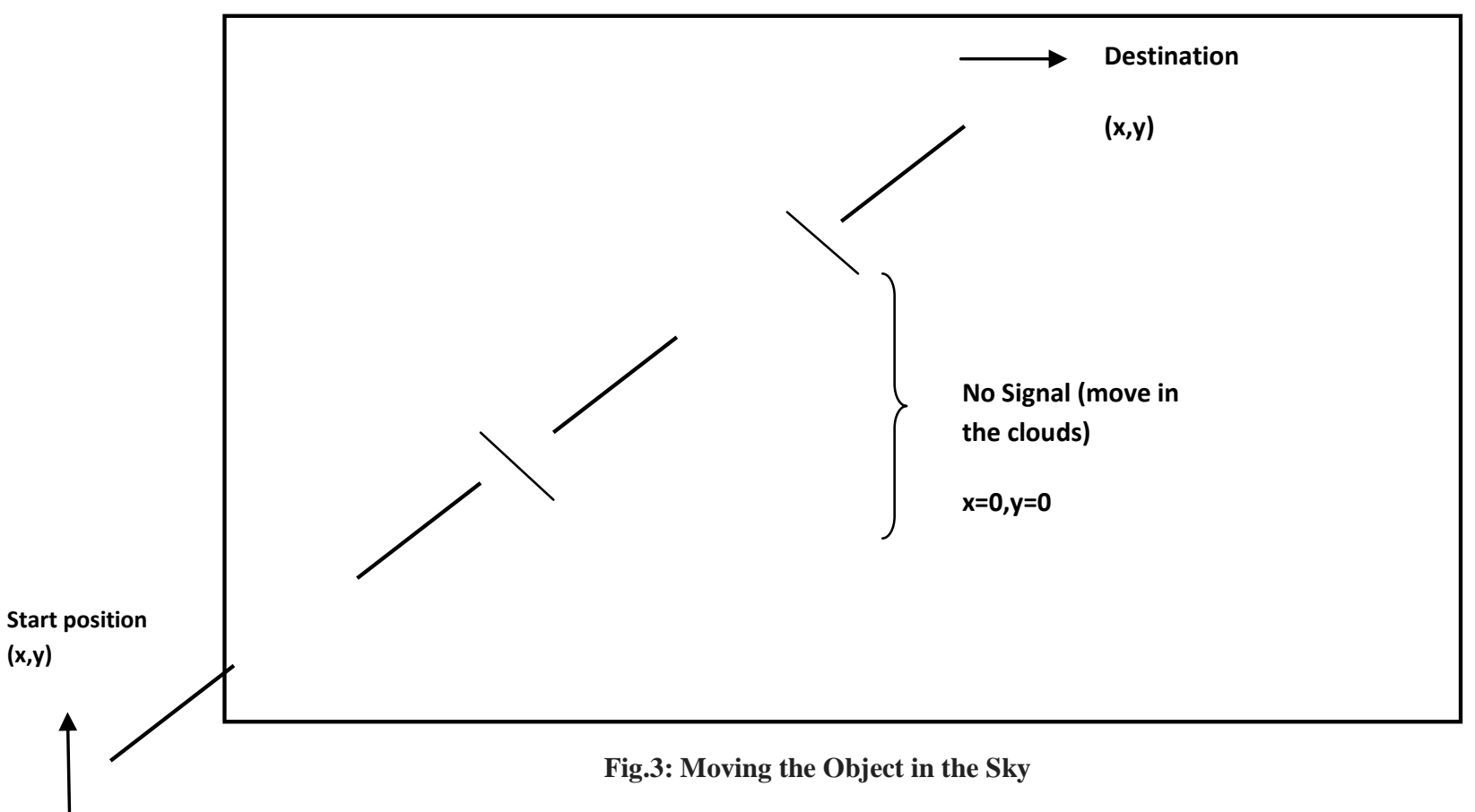


The Algorithm- 4 describe the details of the object moving in the different location in some of the duration there is no signal indication of the remarks, in that situation the Value of the object coordinate, the speed and nop everything will indicate zero value. To find the $\mathrm{x}$ and $\mathrm{y}$ coordinate, speed and nop using the count time from the start duration of the no signal point to end duration. The step 1 compares the object value with the destination point, continue the loop until it reach the destination, if the object $\mathrm{x}$ and $\mathrm{y}$ coordinates is zero indicate the object moving in the no signal location. The time only increases in that location, the remarks is set as no signal. The step2 update all the attributes value until the object goes to the signal indication ( $\mathrm{x}$ and $\mathrm{y}$ coordinate value).

\section{Algorithm-4: Object1 (int x, int y)}

Variables: $x, y$ are the coordinate value of the object1, set the timer interval, object1 start the movement from the direction left to right, set the destination(d) value of the object1.

Move to step2

endif

2) Save all the values and remarks in the database until the value reaches the destination.

3) Exit

The table(2) it contains the values of the each pixel movement of x_coordinate,y_coordinate,speed,nop,counttime(ctime) and remarks the place of object move on no signal point area.

\section{Calculate the distance and Speed}

$$
\begin{aligned}
& \text { Distance }_{\text {nosignal }} \text { : } \\
& \mathrm{X} \_ \text {cor }=\mathrm{X}_{\mathrm{e}}-\mathrm{X}_{\mathrm{s}} \\
& =\left[\text { Distance }_{\text {final }}\left(\mathrm{X}_{\mathrm{s}}\right)-20\right]-\left[\text { Distance }_{\text {init }}\left(\mathrm{X}_{\mathrm{e}}\right)+15\right] \\
& =7710-6497=1213 \text { pixels } \\
& \mathrm{y}_{-} \text {cor }=\mathrm{Y}_{\mathrm{e}}-\mathrm{Y}_{\mathrm{s}} \\
& =\left[\text { Distance }_{\text {final }}\left(\mathrm{Y}_{\mathrm{s}}\right)-20\right]-\left[\text { Distance }_{\text {init }}\left(\mathrm{Y}_{\mathrm{e}}\right)+15\right] \\
& =92-4477=4385 \text { pixels }[\text { not mention the negative] }
\end{aligned}
$$

Here $y_{\text {_cor }}>\mathrm{x} \_$cor value, so the distance is covered $\mathbf{=} \mathbf{4 3 8 5}$ pixels

$$
\begin{aligned}
\text { Duration }_{\text {nosignal }} & =\text { Etime }- \text { Stime } \\
& =14460-6120=\mathbf{8 3 4 0} \mathbf{~ m s e c}
\end{aligned}
$$

i.e., The speed is calculated each two millisec the distance cover one pixel so 2:1

\begin{tabular}{|c|c|c|c|c|c|c|}
\hline OName & $\mathbf{X}$ & $\mathbf{Y}$ & Speed & NOP & CTime & Remarks \\
\hline Obj1 & 0 & 0 & 0 & 0 & 6120 & $\begin{array}{c}\text { No } \\
\text { Signal }\end{array}$ \\
\hline Obj1 & 0 & 0 & 0 & 0 & 6150 & $\begin{array}{c}\text { No } \\
\text { Signal }\end{array}$ \\
\hline Obj1 & 0 & 0 & 0 & 0 & 6180 & $\begin{array}{c}\text { No } \\
\text { Signal }\end{array}$ \\
\hline Obj1 & 0 & 0 & 0 & 0 & 6210 & $\begin{array}{c}\text { No } \\
\text { Signal } \\
\end{array}$ \\
\hline Obj1 & 0 & 0 & 0 & 0 & 6240 & $\begin{array}{c}\text { No } \\
\text { Signal }\end{array}$ \\
\hline Obj1 & 0 & 0 & 0 & 0 & 6270 & $\begin{array}{c}\text { No } \\
\text { Signal }\end{array}$ \\
\hline Obj1 & 0 & 0 & 0 & 0 & 6300 & $\begin{array}{c}\text { No } \\
\text { Signal }\end{array}$ \\
\hline Obj1 & 0 & 0 & 0 & 0 & 6330 & $\begin{array}{c}\text { No } \\
\text { Signal } \\
\end{array}$ \\
\hline Obj1 & 0 & 0 & 0 & 0 & 6370 & $\begin{array}{c}\text { No } \\
\text { Signal }\end{array}$ \\
\hline .. &.. &.. & .. & .. & .. & .. \\
\hline Obj1 & 0 & 0 & 0 & 0 & 14460 & $\begin{array}{c}\text { No } \\
\text { Signal }\end{array}$ \\
\hline
\end{tabular}

1) If the object1 value compare with the destination (denote as d) value.

If object1.x<d.left and object1.y>d.top then

For $\mathrm{i}=$ object $1 . \mathrm{x}$ to d.left

If object $1 . x=0$ and object $1 . \mathrm{y}=0$ then

$$
\begin{aligned}
& \mathrm{x}_{\mathrm{i}}=0 \\
& \mathrm{y}_{\mathrm{i}}=0 \\
& \text { nop }=0 \\
& \text { speed }=0 \\
& \text { tcount=tcount+interval } \\
& \text { Remarks="no signal" }
\end{aligned}
$$

Table.2. Individual report for the object move in the sky no signal point

OName - Object Name(Obj1);X,Y-Object position in $\mathrm{x}$ and y coordinate pixel values; NOP-Number of pixel per msec;CTime-Count time for each time object movement.

In the above calculation ratio of the duration and distance, $\mathrm{Xe}, \mathrm{Xs}, \mathrm{Ye}$ and $\mathrm{Ys}$ is the maximum and minimum value of the $\mathrm{x}$ and $\mathrm{y}$ coordinates. Etime, Stime is the ending and starting time duration. In the no signal stage is calculated the value based on the ending value based on number of pixel (nop) value of the before no signal value and ending of no signal pixel value i.e nop value, the ratio is $2: 1$. The value of nosignal only way find in the previous and after value of the remarks of the database. Here the database table (2) to keep the entire value object attributes in dynamic manner. 


\section{COMPARATIVE STUDY}

The table3 shows the details of comparison diagram of existing and proposed work.

Table.3.Comparative Study of Existing and Proposed Work

\begin{tabular}{|l|l|l|}
\hline Sl.No & Existing Work & Proposed \\
\hline 1. & $\begin{array}{l}\text { Object using point } \\
\text { and balloon }\end{array}$ & $\begin{array}{l}\text { Object using } \\
\text { point, line or may } \\
\text { be fetch any } \\
\text { object }\end{array}$ \\
\hline 2. & $\begin{array}{l}\text { Region using } \\
\text { Standard }\end{array}$ & $\begin{array}{l}\text { Using different } \\
\text { Region }\end{array}$ \\
\hline 3. & $\begin{array}{l}\text { Not included } \\
\text { refection }\end{array}$ & $\begin{array}{l}\text { Reflection } \\
\text { Included }\end{array}$ \\
\hline 4. & $\begin{array}{l}\text { Not included } \\
\text { Hiding position of } \\
\text { object }\end{array}$ & Hiding \\
\hline
\end{tabular}

\section{SAMPLE SQL QUERY}

The GROUP BY commands is used to grouping the same type objects or remarks. The aggregate values are min, max values are used to display the result.

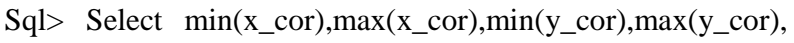
$\max$ (speed), $\max ($ nop), $\min ($ ctime), $\max$ (ctime), $\max$ (remarks) from $\mathrm{m}_{-}$sky group by remarks;

Sql> select $\min ($ left), $\max ($ left), $\min ($ top), $\max ($ top), $\max$ (speed), $\max ($ nop), $\min ($ ctime), $\max$ (ctime), $\max ($ remarks)from $\mathrm{m} \_$water1 group by remarks having remarks like ?

\section{CONCLUSION}

In this research discussed the object movement in the three different environments, the object move in the different location, speed and timing. In the algorithm and equations are described information about object movement finding in different speed, position and hiding values. The remarks attribute easily to identify the object current movement. The above movement of object considered based on the screen pixel values and timing of milliseconds. The database used to store and retrieve entire movement of the object with effective query analyzation. In future research extend the concept implement in the real time data, the reflection part is maximum elaborative manner, the direction of flow in different covered in all the direction and hiding position finding in very accurate also the algorithmic development substitute real time values.

\section{REFERENCES}

[1] Jose Antonio Cotelo Lema, Luca Forlizzi, Ralf Hartmut Guting, Enrico Nardelli and Markus Schneider, " Algorithms for Moving Objects Databases", the computer journal, 46(6), (C) British Computer Society 2003,pp-1 to 33 .

[2] Michael F. Worboys "A Unified Model for Spatial and Temporal Information”, The Computer Journal, Vol 37, no. 1, pp25-34, 1994.

[3] Reasey Praing \& Markus Schneider, "Modeling Historical and Future Spatio-Temporal Relationships of Moving Objects in Databases", University of Florida,
Department of Computer \& Information Science \& Engineering, 2007

[4] Markus Schneider, "Moving object in database and GIS: State of the art and open problem" pp.171-186, 2008.

[5] Hoda M.O.Mokhtar "HITS: a history Based intelligent transportation system”, IJDKP vol.1, No.2, March 2011.

[6] Ganesh Viswanathan \& Markus Schneider, "The object interaction graticlue for cardinal direction querying in moving objects data warehousing", NASA-AIST-080081 .

[7] Dieter Pfoser, "Issues in the Management of Moving Point Objects”,Ph.D thesis, Aalborg University,2009.

[8] Yufei Tao, Dimitris Papadias, “Time-Parameterized Queries in Spatio-Temporal Databases", ACM SIGMOD'2002, June 4-6, Madison, Wisconsin, USA.

[9] Ghazi H. Al-Naymat, "New Methods foe Mining Sequential and Time Series Data", Ph.D Thesis, The University of Sydney, June 2009.

[10] Daniel B. Neill "Detection of Spatial and SpatioTemporal Clusters",Ph.D thesis, Carnegie Mellon University,2006.

[11] Dieter Pfoser, "Indexing the Trajectories of Moving Objects”,Data Engineering,June2002, Vol. 25 No. 2, IEEE Computer Society,pp.3-9.

[12] T.Abraham and J.F.Roddick, "Survey of Spatiotemporal Databases", Research Report CIS-96011,School of Computer and Information Science, University of South Australia, 1996.

[13] Ralf Hartmut Gutingand Markus Schneider "Moving Objects Databases", Elsevier publication, 2005.

[14] H. M. Abdul-Kader "Location Updating Strategies in Moving Object Databases", International Journal of Computer Theory and Engineering, Vol. 1, No. 1, April 2009.

[15] Xiangyu Ye, "An Indexing Structure and Application Model for Vehicles Moving on Road Networks", Florida International University, dissertation, 2004.

[16] Arif Khan \& Markus Schneider, "Topological reasoning between complex regions in database with frequent updates", ACM GIS' 10, Nov 2-5, 2010. San Jose, CA, USA.

[17] Hechen Liu \& Markus Schneider "Querying moving objects with uncertainty in spatio-temporal databases", 2010

\section{AUTHORS PROFILE}

Dr.V.Karthikeyani received her $\mathrm{PhD}$ (computer science) in 2007 from Periyar University.MCA from Madras University in 1995 and B.Sc (mathematics) from Madurai Kamaraj University in 1992.she is 16 years of teaching experience in various engineering and arts \& science colleges. She was Published 10 papers in National and International Journals .she is a Life member in ISTE, CSI and ACM-CSTA. Her Research interest is Image Processing, Multimedia, Data Mining and Computer Graphics. 
I.Shahina Begam received her M.Phil(computer science) from Bharathidasan university in 2005,MCA from periyar University in 2002. BES(electronic science) from Madras University in 2001. She is working in the post of Assistant Professor totally eight years. She was published two international journals. She is interests in the area of database management system, moving object database, data warehousing and data mining.

K.Tajudin received his M.Phil(computer science) from Bharathidasan university in 2005,M.S(information technology and management) from Bharathidasan university in 2003 and B.Sc(chemistry) from Madras
University in 2001. He is working in the post of Assistant Professor in totally four years. $\mathrm{He}$ was published two international journals. He is interest in the area of computer network, data mining and database.

I.Parvin Begum received her M.Phil(computer science) from Periyar University at Salem, in 2007,MCA from Madurai Kamaraj University in 2004. BES (electronic science) from Madras University in 2001. she is working in the post of lecturer in totally four years. She was published one international journals. She is interest in the area of computer Graphics, data mining and database. 